\title{
Construyendo capacidades tecnológicas en escenarios inestables: empresas manufactureras argentinas y brasileñas
}

\author{
Anabel Marin, Lilia Stubrin y María Amelia Gibbons
}

RESUMEN

Desde la década de 1970, el contexto macroeconómico en la Argentina y el Brasil se caracterizó por cambios económicos drásticos e inestabilidad. En numerosos estudios se ha documentado el efecto generalmente negativo de ese entorno en las capacidades de innovación del sector manufacturero. En este trabajo, en cambio, se analiza el posible surgimiento de nuevas capacidades de innovación en dicho período, revelándose dos fenómenos importantes. Primero, un número no menor de empresas, incluso en entornos inestables, redoblaron sus esfuerzos innovadores. Segundo, estas empresas se concentran en un grupo reducido de sectores relacionados con las ventajas estáticas de estos países o en sectores favorecidos por regímenes sectoriales específicos. Los resultados encontrados, aunque exploratorios, contribuyen a la discusión sobre la construcción de capacidades innovadoras en contextos macroeconómicos inestables y la capacidad de los sectores vinculados a las ventajas estáticas de ambos países para generar espacios de innovación y creación de valor.

PALABRAS CLAVE

CLASIFICACIÓN JEL

AUTORAS
Industria, productos manufacturados, condiciones económicas, innovaciones tecnológicas, investigación y desarrollo, datos estadísticos, Argentina, Brasil

$\mathrm{O} 14,025, \mathrm{O} 32$

Anabel Marín es investigadora del Consejo Nacional de Investigaciones Científicas y Técnicas (conicet), el Centro de Investigaciones para la Transformación (CENIT) y la Universidad Nacional de Tres de Febrero (UNTREF), Argentina. a.i.marin@fund-cenit.org.ar

Lilia Stubrin es becaria posdoctoral del Centro de Investigaciones para la Transformación (CENIT), Argentina. stubrin@merit.unu.edu

María Amelia Gibbons es asistente de investigación del Centro de Investigaciones para la Transformación (CENIT) y asistente de docencia en la Universidad de San Andrés, Argentina. amelygibb@gmail.com 


\section{I}

\section{Introducción}

En este trabajo se estudia la experiencia de empresas manufactureras argentinas y brasileñas en la acumulación de capacidades innovadoras a lo largo de las últimas dos décadas. El marco temporal elegido coincide con un contexto de inestabilidad en la economía y de cambios en las políticas económicas en ambos países. Esto es poco común en estudios acerca de la construcción de capacidades innovadoras en países emergentes, trabajos en que se han analizado períodos largos de continuidad acumulativa que abarcan dos, tres o más décadas ${ }^{1}$. En tales estudios se evidencia una continuidad de largo plazo en dos niveles. Por una parte, las trayectorias de desarrollo de capacidades a nivel microeconómico generalmente avanzaron sin complicaciones por etapas sucesivas de actividades innovadoras cada vez más "profundas". Por otra, dentro de los relativamente pocos países asiáticos donde se generó la mayor parte de los conocimientos sobre el comportamiento tecnológico a largo plazo de las empresas industriales en países emergentes, los contextos institucionales y de política tuvieron una evolución continua y una estabilidad relativa. Aunque existieron cambios en los énfasis principales de las políticas y ciertas perturbaciones en el ámbito político y económico, las crisis no fueron tan amplias ni perjudiciales como para destruir y dispersar las capacidades innovadoras existentes. Por lo tanto, las principales trayectorias del desarrollo tecnológico rara vez fueron perturbadas y, menos aún, truncadas o invertidas.

La experiencia latinoamericana es muy diferente. Luego del período de construcción acumulativa de capacidades a nivel de empresa, que se produjo en el contexto de los regímenes y políticas relativamente estables de la época de la industrialización mediante sustitución de importaciones (ISI), entre los años cincuenta y setenta

\footnotetext{
${ }^{1}$ Por ejemplo, Amsden (1989) en la República de Corea, entre los años sesenta y ochenta; Kim (1997) en la República de Corea sobre trayectorias cumulativas; Hobday (1995) en Singapur, la provincia china de Taiwán y la República de Corea en los 20 a 30 años posteriores a la década de 1970; o Mathews y Cho (2000) en su estudio del desarrollo de la industria semiconductora en países asiáticos.
}

del siglo pasado, la inestabilidad y las fluctuaciones en el ciclo económico se volvieron recurrentes. Por una parte, agudas crisis financieras y económicas afectaron a la mayoría de los países de la región. Por otra, los países implementaron profundos cambios en la orientación de la política económica en las últimas décadas. Entre las décadas de 1980 y 1990 se liberalizaron el comercio y las finanzas, se redujo la participación del Estado en la economía, se apreció la moneda y se favorecieron inversiones extranjeras. Posteriormente, el Estado fue recuperando injerencia en la economía y las políticas neoliberales comenzaron a retraerse.

Estas fluctuaciones y los cambios bruscos en el contexto político-económico forman el centro de la presente investigación sobre la acumulación de capacidades tecnológicas en la región, tomando como estudios de caso la industria manufacturera en el Brasil y la Argentina ${ }^{2}$. Anteriormente, en muchos trabajos se ha caracterizado el patrón de innovación de las empresas en los países latinoamericanos durante las últimas décadas (entre otros: Katz, 2001 y 2007; Cimoli y Katz, 2003; Chudnovsky, López y Pupato, 2006; Baer, 1970 y 1972; Teitel y Thoumi, 1987; Erber, Guimarães y Tavares Araújo Jr., 1974). En la mayoría de estos estudios se ha analizado una repercusión generalmente negativa de las reformas económicas de las décadas de 1980 y 1990 en la capacidad de innovación del sector manufacturero ${ }^{3}$. Sin embargo, en este trabajo se adopta una perspectiva algo distinta. Cabe preguntarse si durante el período de drásticos cambios económicos y escasa estabilidad macroeconómica —en el que primó un efecto negativo en las capacidades innovadoras de las empresas- ha habido algún proceso

\footnotetext{
${ }^{2}$ Un antecedente de este trabajo puede encontrarse en Marín y Bell (2012), centrado en el caso argentino en el período 1992-2001.

${ }^{3}$ Aunque también se rescataron algunos efectos positivos derivados de las actividades de innovación realizadas. En particular, los incrementos en productividad y la reducción de la brecha de eficiencia con países líderes como resultado de la inversión en tecnología en varias áreas de la economía, sobre todo en industrias vinculadas a la explotación y el procesamiento de los recursos naturales (RRNN).
} 
de generación de nuevas capacidades de innovación en el sector manufacturero, y cuál es la ecología sectorial de ese proceso y en qué tipo de empresas surgieron esas capacidades. El interés de la presente investigación se focaliza en las nuevas aglomeraciones de empresas innovadoras que se crearon luego de las transformaciones estructurales experimentadas en estos países durante los años noventa. Conviene aclarar que el enfoque es dinámico y relativo, no estático ni absoluto. El interés no recae en casos individuales de empresas exitosas en un punto del tiempo, sino más bien en cómo han evolucionado los esfuerzos y resultados tecnológicos de las principales aglomeraciones de empresas que innovan.

El análisis empírico se basa en evidencia provista por las encuestas de innovación. En la Argentina, las encuestas disponibles cubren el período comprendido entre principios de los años noventa y mediados de la década de 2000. En el Brasil, en cambio, las encuestas disponibles cubren un período de tiempo más corto, concentrándose en la década antes mencionada. El análisis se centra en las empresas que, en los diferentes países y períodos analizados, estaban a la vanguardia de la actividad innovadora. Para la identificación de estas empresas se consideró a aquellas que dicen haber introducido una innovación de producto, proceso o de ambos cuyo carácter fue novedoso para la economía mundial al inicio del período estudiado. Este indicador de resultado del proceso de innovación, aunque de carácter subjetivo, permite identificar el extremo más significativo de la distribución de empresas innovadoras.

La evidencia analizada permite ver algunos hechos llamativos (o no del todo esperables). En primer lugar, se observa que parecieran estar emergiendo nuevas aglomeraciones de empresas innovadoras, a pesar de las crisis y de los cambios profundos en la orientación de la política económica que han caracterizado a estos países en las últimas décadas. Esta evidencia, aunque preliminar, objeta una visión pesimista en la región que ha identificado mayormente resultados negativos. Resulta interesante, sin embargo, constatar que estos conglomerados de innovadores se hallan en un reducido número de sectores protegidos por ventajas estáticas de localización — como aquellos vinculados (directamente o indirectamente) a los recursos naturales (RRNN) o sectores tradicionales - e incluso favorecidos por políticas públicas, como es el caso del sector automotriz que goza de un régimen especial de protección tanto en el Brasil como en la Argentina. Finalmente, y cuestionando una vez más las visiones pesimistas acerca de la intensidad innovadora y el potencial dinamismo de los sectores protegidos por ventajas estáticas (como aquellos vinculados a los RRNN y los sectores tradicionales), se advierte que las empresas en estos sectores, aunque parten de niveles muy bajos, están realizando esfuerzos significativos, mayores que el promedio, para mejorar su desempeño innovador y tecnológico.

Es necesario aclarar que la presente investigación es de carácter netamente exploratorio. No se intenta dar una respuesta definitiva a todos los interrogantes planteados, sino más bien aportar nueva evidencia empírica a la discusión acerca de la generación de ventajas de innovación en los países de la región. La información analizada no cubre los últimos años, ya que después de 2005 no están disponibles las encuestas de innovación. Sin embargo, esto no les resta importancia a las principales conclusiones de este trabajo, ya que, en gran parte, los fenómenos identificados tienen repercusiones de más largo plazo. Por último, cabe destacar que el objetivo del trabajo es presentar a la Argentina y el Brasil como dos estudios de caso, pero no establecer una comparación entre ambos países. La dificultad de efectuar una comparación radica en que se trata de distintas muestras de empresas diferentes tomadas para períodos disímiles, y que el indicador utilizado para identificar a las empresas innovadoras, por ser subjetivo, puede estar influido por sesgos nacionales en la manera en que se interpreta y capta la información empleada en el indicador.

La estructura del artículo es la siguiente. En la sección II se describe brevemente el contexto de inestabilidad en que las empresas manufactureras de la Argentina y el Brasil han operado luego del período relativamente estable de protección durante la ISI. En la sección III se exponen los datos y la metodología. En la sección IV se presenta el primer conjunto de resultados, identificándose los sectores a los que pertenecen las empresas más innovadoras. La sección $\mathrm{V}$ se centra en la caracterización de las empresas especialmente innovadoras, identificando sus orígenes y antecedentes, y sus patrones de comportamiento tecnológico. En la sección VI se presentan las implicaciones y se ofrecen algunas reflexiones a partir de los resultados encontrados. 


\section{II}

\section{El contexto: de la protección a la desprotección en un escenario de profunda inestabilidad}

\section{Los inicios de la industria: etapa proteccionista}

El proceso de desarrollo industrial y tecnológico de la industria manufacturera en la Argentina y el Brasil se inició casi espontáneamente a fines del siglo XIX, cuando el modelo agroexportador dominaba en ambos países. Sin embargo, a partir de la década de 1930, el Estado comenzó a impulsar activamente el proceso de industrialización por medio de políticas de protección del mercado interno (es decir, barreras arancelarias), la creación de instituciones estatales (esto es, instituciones de crédito, bancos de desarrollo) y la provisión de infraestructura. Como resultado de estas políticas, cuatro décadas después, la Argentina y el Brasil habían desarrollado un sector manufacturero diversificado y de cierta sofisticación, que había empezado a operar como motor de estas economías.

El proceso de industrialización en la Argentina y el Brasil, sin embargo, mantuvo desde sus inicios una alta dependencia de insumos y bienes de capital importados. Esta característica, en conjunto con el débil crecimiento de las exportaciones, se tradujo en recurrentes déficits en la balanza de pagos. Ello repercutió en las principales variables macroeconómicas generando crisis regulares, inestabilidad e inflación.

Para muchos autores, la incapacidad del sector manufacturero de generar saldos exportables, reducir la dependencia de las importaciones y garantizar un proceso de crecimiento sin sobresaltos estuvo especialmente vinculada a ciertas limitaciones y desequilibrios que caracterizaron al proceso de aprendizaje tecnológico y de acumulación de capacidades industriales (Katz, 1972, 1987, 2001 y 2007; Katz y Kosacoff, 1989 y 2000; Dahlman, 1984; Dahlman y Fonseca, 1987; Naciones Unidas, 1969). Se argumenta que hubo un crecimiento relevante en el sector manufacturero durante este período, que estuvo acompañado de incrementos sostenidos en la productividad agregada y de significativos procesos de aprendizaje tecnológico, sobre todo en algunos sectores de baja y media tecnología (Teitel y Thoumi, 1987).
Sin embargo, existieron dos limitaciones importantes a este proceso. Primero, la excesiva integración vertical y la limitada especialización en ingeniería -en combinación con la oferta abundante de mano de obra calificada- condujeron a un esfuerzo doméstico por adaptar tecnologías y mejorarlas de manera incremental, que fue excesivo con respecto a las inversiones en cambios tecnológicos incorporados o a la realización de actividades de investigación y desarrollo (I+D) para reducir significativamente los costos y desarrollar productos nuevos e innovaciones más "radicales" (Teitel, 1981; López, 1996). De hecho, la evidencia indica que los gastos en $\mathrm{I}+\mathrm{D}$, derechos de autor, patentes y concesiones de franquicias fueron bajos en relación con los parámetros internacionales de la época (Katz, 1972). Segundo, la difusión de tecnologías hacia adentro de los sectores fue muy limitada (Dahlman y Frischtak, 1990). En cada sector generalmente coexistían, por una parte, un conjunto limitado de empresas que lograron alcanzar estándares mundiales de productividad mediante esfuerzos de innovación en ingeniería, calidad, diseño y organización; y por otra, el grueso de las firmas que se caracterizaban por mostrar bajos niveles de productividad, escasos esfuerzos de innovación, equipamiento obsoleto y productos de baja calidad respecto de los estándares internacionales. Se argumenta que la falta de competencia externa fue un factor decisivo para desincentivar la modernización tecnológica y la innovación en gran parte del espectro productivo.

\section{Reformas estructurales: destrucción y resurgimiento}

El progresivo proceso de industrialización que, con altibajos, se desarrolló a lo largo del siglo XX se frenó abruptamente a mediados de los años setenta con la apertura de la economía y la eliminación de regulaciones y subsidios que protegían al sector industrial (Katz y Kosacoff, 2000). Además de ello, la inestabilidad económica y la crisis de la deuda en la década de 
1980 acentuaron aún más el proceso de estancamiento económico e industrial. Como consecuencia de estos cambios contextuales y de política, a fines del decenio de 1970 y principios de los años ochenta, la industria manufacturera sufrió en la Argentina y el Brasil la crisis más profunda en la historia del sector industrial. Esta crisis se manifestó, entre otros fenómenos, en la salida de empresas del mercado, la abrupta caída de la inversión y una creciente obsolescencia del capital invertido (véanse, entre otros, Katz, 2001; Kosacoff, 1996; Ferraz, Kupfer y Serrano, 1999, y Baer, 2001).

En la década de 1990 se profundizó el proceso de liberalización y desregulación iniciado a mediados de los años setenta, aunque también emergieron algunos elementos novedosos: la privatización de la mayoría de las empresas públicas, la estabilización de los precios ${ }^{4}$ y la apreciación del tipo de cambio. Estos elementos impusieron un nuevo entorno competitivo a la industria. En los estudios que se hicieron a finales de los años noventa y principios de la década de 2000 se identificaron las siguientes tendencias como consecuencia de las transformaciones llevadas a cabo en el decenio de 1990:

i) Desaceleración del crecimiento industrial: en la Argentina, el sector industrial redujo su contribución al producto interno bruto (PBI) del 19\% en 1990 al $16 \%$ en 2000; mientras que en el caso brasileño la industria aminoró su tasa de expansión manteniendo su participación en un nivel similar a lo largo de la década de 1990 (Barros de Castro, 2003; Ferraz, Kupfer e Iootty, 2004).

ii) Cambios a nivel sectorial: se retrajeron algunas industrias ${ }^{5}$, se agregaron algunas nuevas a la matriz industrial y otras desaparecieron. Los productos básicos (industriales y basados en RRNN) ganaron mayor peso relativo (Cimoli y Katz, 2003; Ferraz, Kupfer e Iootty, 2004).

iii) Aumento en la heterogeneidad y la concentración: algunas empresas, en su mayoría grandes firmas y filiales de multinacionales, aumentaron sustancialmente sus niveles de productividad e

\footnotetext{
${ }^{4}$ La estabilización de los precios a partir del establecimiento de la ley de convertibilidad en la Argentina en 1991 y del Plan Real en el Brasil en 1994.

${ }^{5}$ En la Argentina, durante la década de 1990 se retrajeron los sectores metalmecánico y químico, las dos industrias más dinámicas durante el período de industrialización mediante sustitución de importaciones (ISI).
}

igualaron a las mejores prácticas internacionales por medio de inversiones en equipos y la implementación de cambios organizacionales (por ejemplo, mediante la incorporación de la automatización de procesos). Sin embargo, una gran cantidad de empresas — sobre todo pequeñas y medianas (pymes) — no pudieron implementar estos cambios y desaparecieron, mientras otras se volvieron simples sobrevivientes logrando mejorar su productividad básicamente a través del despido de empleados (Kosacoff, 1996, 2000a y 2000b; Ferraz, Kupfer e Iootty, 2004; Katz y Bercovich, 1993).

iv) Cierre de la brecha de productividad: en muchos sectores se produjo un proceso de modernización basado en la racionalización de costos y la adquisición de tecnologías desde el exterior. Los canales preferidos pasaron a ser las vinculaciones con proveedores internacionales, la adquisición de licencias y la ayuda técnica en línea. Paralelamente, perdieron peso relativo los proveedores locales (de equipos y empresas de ingeniería) y los laboratorios domésticos de I+D (Katz, 2001; Ferraz, Kupfer y Haguenauer, 1996; Ferraz, Kupfer e Iootty, 2004).

v) Transnacionalización: la Argentina y el Brasil se convirtieron en los principales destinatarios de la inversión extranjera directa (IED) en América Latina. En la Argentina, la participación extranjera en las ventas totales de las 200 más grandes empresas manufactureras aumentó del 43\% en 1994 al 69\% en 1998 (Kulfas, Porta y Ramos, 2002). En el Brasil, entre las 300 empresas más grandes del país, las multinacionales incrementaron su participación en las ventas del 14,8\% al 36,4\% (Rocha y Kupfer, 2002). El movimiento de capital se dirigió principalmente hacia la adquisición de bienes existentes, a través de la privatización, las fusiones y las adquisiciones. En consecuencia, la incorporación de capital extranjero repercutió más en la compra de empresas existentes que en el aumento del capital bruto fijo.

Las características anteriores tuvieron lugar en un marco de gran inestabilidad macroeconómica: períodos de estabilización y crecimiento, seguidos por otros de tasas de crecimiento negativas, impactos de las crisis internacionales y vulnerabilidad externa. La parte principal del presente análisis se centra en estudiar la generación de capacidades innovadoras en el sector manufacturero desde el decenio de 1990 hasta mediados de la década siguiente. 


\section{III}

\section{Datos y métodos}

\section{Datos}

Los datos utilizados surgen de las encuestas de innovación realizadas a las empresas manufactureras en la Argentina y el Brasil. En el caso de la Argentina, se utilizaron las encuestas que abarcan los períodos 1992-1996 y 2002-2004; mientras que en el del Brasil se analizaron las encuestas correspondientes a los años 2000 y 2005. Debido a que la muestra de empresas se modifica entre una encuesta y otra ${ }^{6}$, los datos se basan en una submuestra de empresas que han sido relevadas en cada una de las encuestas consideradas en cada país. En el caso de la Argentina, la submuestra abarca 608 empresas y en el del Brasil, 3.890 empresas.

\section{Método: la selección de las empresas innovadoras}

En el estudio se procura identificar a las empresas que estaban en la vanguardia de la actividad innovadora en el sector manufacturero en la Argentina y el Brasil en las últimas décadas. Es decir, de las 608 empresas encuestadas en la Argentina y de las 3.890 en el Brasil, aquel subgrupo que, en algún sentido, pueda ser catalogado como "particularmente innovador". Se consideró que no es apropiado emplear con este propósito ninguno de los dos indicadores más utilizados para medir la capacidad de innovación de las empresas, esto es, las patentes o los gastos en I+D.

- Los indicadores de resultados basados en patentes por lo general tienen limitaciones, dado que reflejan la actividad inventiva, no innovadora, y porque poseen un sesgo sectorial importante (Scherer, 1983; Harabi, 1995; Levin y otros, 1987). Los indicadores basados en patentes son, además, particularmente poco adecuados en contextos como los de la Argentina y el Brasil, donde las empresas rara vez utilizan patentes para proteger sus nuevos conocimientos. Ello obedece sobre todo a dos razones: i) generalmente las empresas

\footnotetext{
${ }^{6}$ En la Argentina, la encuesta de innovación 1992-1996 cubre a 2.430 firmas y la segunda encuesta (2002-2004) abarca 1.690 empresas. Para el caso del Brasil, las encuestas de innovación de 2000 y 2005 cubren 10.328 y 12.172 empresas, respectivamente.
}

tienen un carácter de adoptantes de tecnologías en la frontera o próximas a esta; ii) el sesgo sectorial que existe en la región hacia industrias de proceso, donde la propensión a patentar suele ser menor. Por ejemplo, en la Argentina, de acuerdo con datos del Instituto Nacional de Estadística y Censos (INDEC), la Secretaría de Ciencia y Tecnología (SECYT) y la Comisión Económica para América Latina y el Caribe (CEPAL), entre 1998 y 2001 solo el 10\% de los innovadores patentaron (Chudnovsky, López y Pupato, 2006). En este estudio, el interés estriba en captar las características generales de todas las empresas innovadoras, no solo de las que patentan, y por ello este tipo de indicadores no resultan del todo adecuados.

- Los indicadores basados en I+D reflejan los esfuerzos realizados por las empresas para crear conocimiento, pero no muestran la eficacia de la actividad y, lo que es más importante en este contexto, tienden a reflejar las diferencias entre las industrias en su intensidad de I+D, en lugar de las diferencias específicas de las empresas en la intensidad de la actividad innovadora. Además, estos indicadores tienden a subestimar las actividades innovadoras estrechamente relacionadas con la producción, así como el procesamiento de la información (Patel, 2000). Sobre la base de la difusión de las encuestas de innovación de muchos países, en los últimos 15 años se han empezado a utilizar otras medidas diferentes para evaluar el carácter innovador de las empresas. La más popular de estas, cuando se trata de medir resultados y no esfuerzos, ha sido el porcentaje de ventas de productos innovadores. En principio, esta es una buena medida directa de los resultados de la innovación, pero discrimina la innovación de procesos (que es particularmente importante en la Argentina y el Brasil). Es por esto que en este trabajo se decidió utilizar otra medida. Siguiendo las distinciones estándares del Manual de Oslo (OCDE, 1997), en las encuestas de innovación se pregunta a las empresas manufactureras sobre el grado de novedad de las innovaciones de producto, procesos o de ambos que introdujeron en el mercado durante el período de la encuesta. Cuando se pregunta a las empresas acerca de su desempeño innovador se ofrecen cuatro tipos de respuestas posibles: 
i) no introdujeron ninguna innovación de productos (o procesos);

ii) introdujeron innovaciones de productos (o procesos) que eran nuevas para la empresa;

iii) introdujeron innovaciones de productos (o procesos) que eran nuevas para la economía local; o

iv) introdujeron innovaciones de productos o procesos que eran novedosas para la economía mundial ${ }^{7}$.

Se considera que una respuesta positiva a la última de estas opciones, en la primera encuesta de innovación realizada en cada país, permite relevar las empresas más innovadoras dentro de este. Una limitación obvia es que las respuestas son subjetivas. Las respuestas de empresas que afirman haber introducido esta categoría de innovación pueden no reflejar de manera muy precisa innovaciones que sean verdaderamente "nuevas para el mercado mundial". Sin embargo, la precisión, en tal sentido, no es aquí el tema principal, ya que la preocupación se orienta principalmente a la capacidad de innovación relativa de las empresas dentro de casa país, y no a identificar líderes mundiales. Las empresas encuestadas tienen la posibilidad de seleccionar maneras de innovar menos novedosas, y pareciera que, al menos en términos relativos, esta categoría expresa adecuadamente el extremo más significativo de la distribución de empresas innovadoras.

En el resto del análisis - para no generar confusión en este sentido - se denominarán como "empresas significativamente innovadoras" aquellas empresas que respondieron haber introducido innovaciones de producto, proceso o de ambos novedosos para la economía mundial. Se hará referencia a las demás simplemente como

${ }^{7}$ Para una explicación de estas categorías, véase la versión más reciente del Manual de Oslo (OCDE, 2005, págs. 57 y 58). “empresas no innovadoras", a pesar de que en este último grupo se incluya a firmas que dicen haber introducido innovaciones que eran nuevas para la empresa o el país. En la muestra de empresas analizadas se encuentra que 68 empresas argentinas (11\%) y 167 (4,3\%) empresas brasileñas son significativamente innovadoras. Entre estas, la mayoría introdujo innovaciones solo en productos (60\% en el Brasil y 67\% en la Argentina). Las firmas que introdujeron en el mercado solo innovaciones de proceso son, en términos relativos, más relevantes en el Brasil $(28,1 \%)$ que en la Argentina $(7,4 \%)$. Y aquellas que introdujeron innovaciones tanto de producto como de proceso representan el 12,6\% en el caso brasileño y el $25 \%$ en el argentino (véase el cuadro 1).

Cabe aclarar que, dado el carácter subjetivo de los indicadores utilizados, los resultados de la Argentina y el Brasil no deberían ser comparados, ya que pueden estar reflejando sesgos nacionales en la manera en que se interpreta la pregunta.

CUADRO 1

\section{Tipos de empresas innovadoras en la Argentina y el Brasil \\ (En cantidad y porcentajes)}

\begin{tabular}{lcc}
\hline Tipo de empresas & Argentina & Brasil \\
\hline No innovadoras & $540(88,8)$ & $3723(95,7)$ \\
Significativamente innovadoras & $68(11,2)$ & $167(4,3)$ \\
Solo innovadoras de procesos & 5 & 47 \\
Solo innovadoras de productos & 46 & 99 \\
Innovadoras de productos y procesos & 17 & 21 \\
Total & $608(100)$ & $3890(100)$ \\
\hline
\end{tabular}

Fuente: elaboración propia sobre la base de la Encuesta sobre la Conducta Tecnológica de las Empresas Industriales Argentinas (1992-1996) y la Encuesta Nacional a Empresas sobre Innovación, Investigación y Desarrollo y Tecnologías de la Información y de las Comunicaciones 2002-2004 en la Argentina; y la Encuesta de Innovación Tecnológica (PINTEC) 2000 y 2005 en el Brasil.

\section{IV}

\section{Análisis 1: distribución sectorial de empresas significativamente innovadoras}

En esta sección se estudia la ecología sectorial de las empresas que se identifican como significativamente innovadoras. En particular, se trata de establecer si existe un conjunto de sectores donde estas empresas se aglomeren, lo que revelará áreas de ventajas comparativas dinámicas. Con ese objetivo se clasificaron los sectores manufactureros (usando la Clasificación Industrial Internacional Uniforme de todas las actividades económicas (CIIU) a tres dígitos) en dos grupos: los sectores que incluyen por lo menos a una empresa significativamente innovadora y los que no. Estos últimos se descartan para el análisis. 
En la Argentina se encuentran 31 sectores con al menos una empresa significativamente innovadora, mientras que en el Brasil son 70 los sectores con esta característica. Cabe destacar, sin embargo, que la distribución de empresas significativamente innovadoras entre sectores dista de ser homogénea. Hay sectores que concentran muchas más empresas significativamente innovadoras que otros (véase la columna 3 de los cuadros 2 y 3 ). Con el propósito de identificar aquellos sectores donde las empresas significativamente innovadoras se aglomeran, se consideró la distribución sectorial de estas firmas y se clasificó a los sectores en dos grupos: i) aquellos con una concentración de empresas que es igual o mayor que la mediana de la distribución ("sectores con aglomeraciones de empresas significativamente innovadoras"), y ii) aquellos que tienen una concentración de empresas inferior a la mediana de dicha distribución ("sectores sin aglomeraciones de empresas significativamente innovadoras") ${ }^{8}$. De aquí en adelante se hace referencia a las empresas del

8 El listado de los sectores sin aglomeraciones de empresas significativamente innovadoras puede ser solicitado a las autoras. primer grupo de sectores como "empresas innovadoras aglomeradas", y a las firmas del segundo grupo de sectores como "empresas innovadoras aisladas".

De los cuadros 2 y 3 surge que las empresas innovadoras aglomeradas se concentran en un conjunto reducido de sectores dentro de la industria manufacturera: en 5 sectores en el caso de la Argentina y en 7 sectores en el caso del Brasil. Estos sectores aglomeran el $42 \%$ y el $31 \%$ de este tipo de firmas en la Argentina y el Brasil, respectivamente.

Con respecto a la composición intrasectorial del grupo de sectores con aglomeraciones de empresas significativamente innovadoras, cabe hacer algunas observaciones adicionales. Las firmas innovadoras aglomeradas representan el $18 \%$ de todas las empresas en esos sectores en la Argentina y el 7\% en el Brasil (véase la cuarta columna de los cuadros 2 y 3 ), es decir, el $82 \%$ y el $93 \%$ de las empresas en los sectores con aglomeraciones de empresas innovadoras no son significativamente innovadoras en la Argentina y el Brasil, respectivamente. Ello sugiere que la selección de este estudio comprende a un grupo relativamente pequeño en el extremo superior de la distribución del carácter innovador de las empresas.

\begin{tabular}{|c|c|c|c|}
\hline Sector & & $\begin{array}{l}\text { Participación de las empresas } \\
\text { significativamente innovadoras } \\
\text { del sector en el total de } \\
\text { empresas significativamente } \\
\text { innovadoras }\end{array}$ & $\begin{array}{l}\text { Participación de empresas } \\
\text { significativamente innovadoras } \\
\text { en el total de empresas del } \\
\text { sector }\end{array}$ \\
\hline (1) & $\begin{array}{c}(2) \\
\text { Empresas } \\
\text { (cantidad) }\end{array}$ & $\begin{array}{c}(3) \\
\text { (en porcentajes) }\end{array}$ & $\begin{array}{c}(4) \\
\text { (en porcentajes) }\end{array}$ \\
\hline Empresas innovadoras aglomeradas & 29 & 42 & 18 \\
\hline $\begin{array}{l}\text { Fabricación de plaguicidas y otros productos químicos } \\
\text { de uso agropecuario }\end{array}$ & 9 & 13 & 17 \\
\hline $\begin{array}{l}\text { Producción, procesamiento y conservación de carne, } \\
\text { pescado, frutas, legumbres, hortalizas, aceites y grasas }\end{array}$ & 7 & 10 & 18 \\
\hline Fabricación de maquinaria agropecuaria y forestal & 5 & 7 & 45 \\
\hline Elaboración de otros productos alimenticios & 4 & 6 & 13 \\
\hline $\begin{array}{l}\text { Fabricación de partes, piezas y accesorios para } \\
\text { vehículos automotores y sus motores }\end{array}$ & 4 & 6 & 24 \\
\hline Empresas innovadoras aisladas & 39 & 58 & 9 \\
\hline Total & 68 & 100 & 11 \\
\hline
\end{tabular}

Fuente: elaboración propia sobre la base de la Encuesta sobre la Conducta Tecnológica de las Empresas Industriales Argentinas (1992-1996). CIIU: Clasificación Industrial Internacional Uniforme de todas las actividades económicas. 


\begin{tabular}{|c|c|c|c|}
\hline Sector & $\begin{array}{l}\text { Empresas } \\
\text { (cantidad) }\end{array}$ & $\begin{array}{l}\text { Participación de las empresas } \\
\text { significativamente innovadoras } \\
\text { del sector en el total de } \\
\text { empresas significativamente } \\
\text { innovadoras } \\
\text { (en porcentajes) }\end{array}$ & $\begin{array}{l}\text { Participación de empresas } \\
\text { significativamente innovadoras } \\
\text { en el total de empresas del } \\
\text { sector } \\
\text { (en porcentajes) }\end{array}$ \\
\hline (1) & $(2)$ & (3) & (4) \\
\hline Empresas innovadoras aglomeradas & 50 & 31 & 7 \\
\hline $\begin{array}{l}\text { Fabricación de productos farmacéuticos, sustancias } \\
\text { químicas medicinales }\end{array}$ & 12 & 7 & 13 \\
\hline Fabricación de calzado y de sus partes & 8 & 5 & 4 \\
\hline $\begin{array}{l}\text { Fabricación de partes, piezas y accesorios para } \\
\text { vehículos automotores y sus motores }\end{array}$ & 7 & 4 & 6 \\
\hline $\begin{array}{l}\text { Fabricación de sustancias químicas básicas, } \\
\text { fertilizantes, compuestos de nitrógeno, plásticos en } \\
\text { formas primarias y de caucho sintético }\end{array}$ & 6 & 4 & 17 \\
\hline $\begin{array}{l}\text { Fabricación de productos químicos de uso } \\
\text { agropecuario }\end{array}$ & 6 & 4 & 24 \\
\hline Fabricación de bombas, compresores, grifos y válvulas & 6 & 4 & 12 \\
\hline Fabricación de productos de plástico & 5 & 3 & 3 \\
\hline Empresas innovadoras aisladas & 97 & 69 & 3 \\
\hline Total & 167 & 100 & 4 \\
\hline
\end{tabular}

Fuente: elaboración propia sobre la base de la Encuesta de Innovación Tecnológica (PINTEC) 2000 y 2005 en el Brasil. CIIU: Clasificación Industrial Internacional Uniforme de todas las actividades económicas.

En términos de la ecología sectorial, una característica relevante por resaltar es que cuatro de los cinco sectores con empresas innovadoras aglomeradas en la Argentina y cuatro de los siete sectores con iguales características en el Brasil se hallan relacionados a través de vínculos de insumo-producto con industrias basadas en RRNN ${ }^{9}$ (véase el cuadro 4). La Argentina concentra la mayor parte de las empresas innovadoras aglomeradas en un conjunto de sectores vinculados directa e indirectamente a los RRNN. Los sectores directamente vinculados a la explotación y procesamiento de RRNN son aquellos que procesan productos agrícolas y ganaderos, mientras que los sectores indirectamente relacionados con la explotación y procesamiento de RRNN son aquellos que proveen insumos a la producción agrícola (fertilizantes y maquinaria). Estos sectores involucran el tipo de actividad productiva en que la Argentina tiene una ventaja competitiva histórica. Conviene destacar, además, que gran parte de la motivación que subyacía al régimen de ISI se basaba en alejar la especialización productiva de este tipo de sectores, entre otras cosas, porque se los consideraba sectores de "baja tecnología" que poco ayudaban a la

\footnotetext{
9 Véase la metodología en el anexo.
}

inserción de actividades innovadoras más importantes dentro de la economía (especialmente en el caso de las industrias más directamente vinculadas a los RRNN).

En el Brasil, los sectores con empresas significativamente innovadoras aglomeradas están más diversificados entre las ramas sectoriales vinculadas y no vinculadas a actividades basadas en la explotación y procesamiento de RRNN. No obstante, más de la mitad de las empresas innovadoras aglomeradas (el 60\%) están relacionadas con actividades basadas en RRNN típicamente como proveedores. Entre los sectores vinculados a los RRNN como proveedores se destacan los de "fabricación de sustancias químicas básicas, fertilizantes, componentes de nitrógeno, plásticos en formas primarias y caucho sintético"; "fabricación de productos agroquímicos"; "fabricación de productos farmacéuticos y sustancias químicas medicinales" y "fabricación de bombas, compresores, grifos y válvulas". Resulta importante aclarar que si bien los sectores recién mencionados aparecen vinculados mediante relaciones insumo-producto a actividades basadas en el procesamiento de RRNN (véase el anexo), sería erróneo asumir que el conjunto total de firmas que pertenecen a dichos sectores esté necesariamente relacionado. Por ejemplo, en el sector de "fabricación de productos farmacéuticos y sustancias 
Clasificación de los sectores con aglomeraciones de empresas significativamente innovadoras en la Argentina y el Brasil

\begin{tabular}{|c|c|c|c|}
\hline & \multicolumn{2}{|c|}{ Sectores vinculados a industrias basadas en RRNN } & \multirow{2}{*}{$\begin{array}{c}\text { Sectores no vinculados a industrias } \\
\text { basadas en RRNN }\end{array}$} \\
\hline & Directamente & Indirectamente & \\
\hline Argentina & $\begin{array}{l}\text { - Producción, procesamiento y } \\
\text { conservación de carne, pescado, } \\
\text { frutas, legumbres, hortalizas, } \\
\text { aceites y grasas (151). } \\
\text { - Elaboración de otros productos } \\
\text { alimenticios (incluidos pan, } \\
\text { galletitas, azúcar, cacao, té, yerba, } \\
\text { y otros) (154). }\end{array}$ & $\begin{array}{l}\text { - Fabricación de plaguicidas y } \\
\text { otros productos químicos de uso } \\
\text { agropecuario (242). } \\
\text { - Fabricación de maquinaria } \\
\text { agropecuaria y forestal (292). }\end{array}$ & $\begin{array}{l}\text { - Fabricación de carrocerías para } \\
\text { vehículos automotores; fabricación } \\
\text { de remolques y semirremolques } \\
\text { (343). }\end{array}$ \\
\hline Brasil & & $\begin{array}{l}\text { - Fabricación de sustancias químicas } \\
\text { básicas, fertilizantes, compuestos } \\
\text { de nitrógeno, plásticos en formas } \\
\text { primarias y de caucho sintético } \\
\text { (241). } \\
\text { - Fabricación de productos químicos } \\
\text { de uso agropecuario (242). } \\
\text { - Fabricación de productos } \\
\text { farmacéuticos, sustancias químicas } \\
\text { medicinales (245). } \\
\text { - Fabricación de bombas; } \\
\text { compresores; grifos y válvulas } \\
\text { (291). }\end{array}$ & $\begin{array}{l}\text { - Fabricación de calzado y de sus } \\
\text { partes (193). } \\
\text { - Fabricación de partes, piezas } \\
\text { y accesorios para vehículos } \\
\text { automotores y sus motores (344). } \\
\text { - Fabricación de productos de } \\
\text { plástico (252). }\end{array}$ \\
\hline
\end{tabular}

Fuente: elaboración propia sobre la base de matriz de insumo-producto (MIP) de la Argentina y el Brasil.

RRNN: recursos naturales.

químicas medicinales" pueden agruparse tanto firmas innovadoras proveedoras de insumos a actividades basadas en RRNN, como otras no vinculadas a los RRNN (por ejemplo, empresas farmacéuticas dedicadas a la salud humana).

Otros sectores que aglomeran empresas innovadoras, pero que no están vinculados a RRNN, son aquellos tradicionales como la "fabricación de calzado y sus partes" y la "fabricación de productos plásticos". Además, surgen empresas innovadoras aglomeradas no vinculadas a RRNN en la Argentina y el Brasil en sectores ligados a la producción automotriz (sectores 343 y 344). Estos reflejan lo que resta de la floreciente industria metalmecánica automotriz que se fomentó durante el período de ISI, y que desde principios de la década de 1990 está en el centro de un sistema de políticas sectoriales que incluye un régimen de comercio especial entre ambos países e incentivos para productores.

Los sectores con aglomeraciones de empresas innovadoras se consideraron más innovadores, dado que este tipo de firmas representan un porcentaje mayor de todas las empresas en esos sectores que en el caso de los sectores sin aglomeraciones de empresas innovadoras (véase la cuarta columna de los cuadros 3 y 4). Sin embargo, cabe subrayar que dentro de los últimos hay varios sectores donde las empresas significativamente innovadoras representan un porcentaje mayor de las empresas sectoriales que en el grupo de firmas con aglomeración de empresas significativamente innovadoras. No obstante, estos sectores comprenden a muy pocas empresas que más bien parecieran entidades aisladas de la actividad innovadora que áreas de aglomeración sectorial. Algunos sectores con estas características son el de "elaboración de combustible nuclear" (100\%) en la Argentina y el Brasil, el de "fabricación de hilos y cables aislados" (50\%) en la Argentina, y el de "actividades de servicios relacionadas con la extracción de petróleo y gas, excepto las actividades de prospección" (33\%) en el Brasil.

En resumen, se constata que las empresas que se identificaron como significativamente innovadoras en los países analizados se aglomeran en un conjunto reducido de sectores. En el caso de la Argentina, cuatro de cinco sectores se encuentran directa o indirectamente vinculados a actividades de explotación y procesamiento de RRNN. En el caso del Brasil hay más variabilidad, no existen sectores directamente vinculados a los RRNN como clientes, pero aparecen algunos sectores claramente relacionados con los RRNN como proveedores, y, lo que es llamativo, también algunos sectores tradicionales. Común 
a ambos casos es la aparición del sector automotriz, que tiene un régimen especial de protección. Dado que tanto las actividades ligadas a los RRNN (sobre todo aquellas identificadas con una vinculación directa) como las tradicionales son consideradas actividades de bajo nivel innovador y agregado de valor, este resultado, por sí solo, es sorprendente. En la sección siguiente esto se aborda en profundidad.

\section{V}

\section{Análisis II: caracterización de las empresas innovadoras aglomeradas}

En esta sección se profundiza acerca de las características de las empresas innovadoras aglomeradas. Interesa en primer lugar entender las características estructurales de estas empresas, tales como el origen de su capital, su tamaño y sus patrones de comportamiento innovador. Con este fin, se comparan las firmas innovadoras aglomeradas con otros grupos de empresas como las innovadoras aisladas, las no innovadoras, las empresas en industrias de "alta tecnología"10 , las filiales de compañías extranjeras y todo el plantel manufacturero en general. Luego, se explora el cambio en el comportamiento innovador de las empresas a lo largo del período estudiado, cuyo comportamiento tecnológico es evaluado a partir de dos tipos de indicadores de esfuerzos de innovación:

i) la intensidad del gasto en I+D de las empresas (gastos en I+D como proporción de las ventas totales); y

ii) la intensidad de la inversión en bienes de capital para la innovación (gastos en bienes de capital como proporción de las ventas totales).

Primero se presenta la evidencia respecto de la Argentina y luego del Brasil. Es importante aclarar que los datos sobre el comportamiento innovador de las empresas en ambos países no son comparables, ya que corresponden a distintos años y fueron recolectados sobre la base de muestras diferentes. La diferencia más relevante es que en el Brasil los promedios se calculan en relación con una muestra de empresas innovadoras (se excluye a las firmas que contestan que no han innovado durante el período), mientras que en la Argentina los promedios se calculan sobre el total de empresas entrevistadas (sean o no innovadoras).

${ }^{10}$ De acuerdo con la clasificación de empresas de alta tecnología de la Organización de Cooperación y Desarrollo Económicos (OCDE).

\section{Las empresas innovadoras aglomeradas en la Argentina}

Las empresas innovadoras aglomeradas vinculadas a los RRNN en la Argentina ${ }^{11}$ son en su mayoría independientes $(52 \%)$ y en menor medida filiales de compañías internacionales (31\%) y grupos empresariales locales (17\%) (véase el cuadro 5). La composición de este conjunto de empresas se diferencia, sin embargo, del total de firmas manufactureras argentinas porque incluye a un menor número de firmas independientes (el $71 \%$ del sector manufacturero está compuesto por empresas independientes), y registra una mayor participación de filiales de compañías extranjeras y de grupos empresariales nacionales (que representan el 17\% y el $11 \%$ del total manufacturero, respectivamente). En cuanto a su tamaño, las empresas innovadoras aglomeradas son, en términos relativos, más grandes que la media del sector manufacturero (véase el cuadro 6). El 48\% de las empresas innovadoras aglomeradas poseen más de 200 empleados, mientras que solo el 39\% del total del plantel de firmas manufactureras posee ese tamaño.

En términos del comportamiento innovador, a principios de la década de 1990 las empresas innovadoras aglomeradas en la Argentina, tomadas en conjunto, realizaron más esfuerzos en inversiones de capital $(1,33 \%)$ que en I+D $(0,10 \%)$. El patrón observado para este tipo de empresas se manifestó también para los otros tipos de firmas considerados (véanse las categorías 2 a 6 del cuadro 7). Sin embargo, la diferencia entre la intensidad de gastos en bienes de capital para la innovación y los

\footnotetext{
11 En principio, en la Argentina el foco está puesto en las empresas aglomeradas en sectores vinculados a RRNN, ya que constituyen la mayor parte.
} 
CUADRO 5

\title{
Empresas en la Argentina por tipo de titularidad
}

(En cantidad y porcentajes)

\begin{tabular}{lcccc}
\hline & $\begin{array}{c}\text { Empresas innovadoras } \\
\text { aglomeradas en industrias } \\
\text { vinculadas a los RRNN }\end{array}$ & $\begin{array}{c}\text { Empresas innovadoras } \\
\text { aisladas }\end{array}$ & $\begin{array}{c}\text { Empresas no innovadoras } \\
\text { en todas las industrias }\end{array}$ & $\begin{array}{c}\text { Total sector } \\
\text { manufacturero }\end{array}$ \\
\hline Grupos empresariales nacionales & 5 & 7 & 57 & $(11)$ \\
Empresas nacionales independientes & $(17)$ & 24 & 39 & $(11)$ \\
Filiales de empresas multinacionales & 15 & $(62)$ & $(73)$ & $(73)$ \\
Total & $(52)$ & $(21)$ & $(16)$ & $(17)$ \\
\hline
\end{tabular}

Fuente: elaboración propia sobre la base de la Encuesta sobre la Conducta Tecnológica de las Empresas Industriales Argentinas (1992-1996) y la Encuesta Nacional a Empresas sobre Innovación, Investigación y Desarrollo y Tecnologías de la Información y de las Comunicaciones 2002-2004 en la Argentina.

RRNN: recursos naturales.

CUADRO 6

\author{
Empresas en la Argentina por tamaño \\ (En cantidad y porcentajes)
}

\begin{tabular}{|c|c|c|c|c|}
\hline & $\begin{array}{l}\text { Empresas innovadoras } \\
\text { aglomeradas en industrias } \\
\text { vinculadas a los RRNN }\end{array}$ & $\begin{array}{c}\text { Empresas innovadoras } \\
\text { aisladas }\end{array}$ & $\begin{array}{l}\text { Empresas no innovadoras } \\
\text { en todas las industrias }\end{array}$ & $\begin{array}{c}\text { Total sector } \\
\text { manufacturero }\end{array}$ \\
\hline Pymes & $\begin{array}{l}15 \\
(52)\end{array}$ & $\begin{array}{c}27 \\
(69)\end{array}$ & $\begin{array}{l}327 \\
(60)\end{array}$ & $\begin{array}{l}369 \\
(61)\end{array}$ \\
\hline Grandes empresas & $\begin{array}{c}14 \\
(48)\end{array}$ & $\begin{array}{c}12 \\
(31)\end{array}$ & $\begin{array}{l}213 \\
(40)\end{array}$ & $\begin{array}{l}239 \\
(39)\end{array}$ \\
\hline Total & $29(100)$ & $39(100)$ & $540(100)$ & $608(100)$ \\
\hline
\end{tabular}

Fuente: elaboración propia sobre la base de la Encuesta sobre la Conducta Tecnológica de las Empresas Industriales Argentinas (1992-1996) y la Encuesta Nacional a Empresas sobre Innovación, Investigación y Desarrollo y Tecnologías de la Información y de las Comunicaciones 2002-2004 en la Argentina.

Nota: pequeñas y medianas empresas (pymes) ( $<200$ empleados) y grandes $(\geq 200$ empleados).

RRNN: recursos naturales.

CUADRO 7

Argentina: comportamiento innovador del sector manufacturero, 1992

Tipo de empresas

\begin{tabular}{cr}
$\begin{array}{c}\text { Intensidad en I+D } \\
\text { Valores medios por empresa } \\
\text { (en porcentajes) }\end{array}$ \\
\hline 0,10 & 1,33 \\
0,01 & 1,49 \\
0,17 & 1,23 \\
0,12 & $-10,16$ \\
0,09 & 1,97 \\
0,20 & 1,57 \\
0,06 & 3,17 \\
0,01 & 1,47 \\
0,06 & 1,81
\end{tabular}

1. Empresas innovadoras aglomeradas en sectores:

- directamente vinculados a los RRNN

- indirectamente vinculados a los RRNN

- no vinculados a los RRNN

2. Todas las demás empresas innovadoras

3. Empresas en industrias "de alta tecnología"

4. Filiales extranjeras

5. Empresas no innovadoras

6. Todas las empresas

0,01

1,47

Fuente: elaboración propia sobre la base de la Encuesta sobre la Conducta Tecnológica de las Empresas Industriales Argentinas (1992-1996) y la Encuesta Nacional a Empresas sobre Innovación, Investigación y Desarrollo y Tecnologías de la Información y de las Comunicaciones 2002-2004 en la Argentina.

RRNN: recursos naturales.

I+D: investigación y desarrollo. 
gastos en I+D fue menor en las empresas innovadoras aglomeradas (tomadas en su conjunto) que para los otros grupos de firmas. Otro dato interesante que surge del análisis, es que las empresas innovadoras aglomeradas junto con aquellas en sectores de alta tecnología son las que más invirtieron en I+D como proporción de sus ventas. Aunque es dable destacar que, en general, los niveles de gasto en I+D fueron bastante magros.

Cuando se analizan separadamente los comportamientos innovadores entre los distintos grupos de empresas aglomeradas, se encuentran diferencias sustanciales entre ellos que vale la pena destacar. La actividad innovadora de las empresas aglomeradas vinculadas a los RRNN como clientes se basó, en mayor medida, en inversiones en nuevas tecnologías que ya estaban incorporadas en los bienes de capital. Los gastos internos en I+D fueron, en cambio, de los más bajos junto con el de las empresas no innovadoras. En cambio, las firmas aglomeradas indirectamente vinculadas a los RRNN, junto con las de alta tecnología, se encuentran entre los tipos de empresas que más invirtieron en I+D a principios de los años noventa. Estas destinaron a actividades de I+D el $0,17 \%$ de sus ventas, mientras que el conjunto de empresas manufactureras invirtió el $0,06 \%$ de sus ventas en ese período.

En cuanto a las empresas innovadoras aglomeradas en industrias vinculadas directamente a los RRNN, este patrón corresponde casi exactamente a las observaciones hechas en estudios previos sobre la actividad innovadora en los años noventa. La innovación consistió, en gran medida, en inversiones intensivas de tecnologías que ya estaban incorporadas en bienes de capital, y como consecuencia se fortaleció la capacidad de producción de manera importante. Sin embargo, casi no había indicios de I+D dentro de las mismas empresas, y la capacidad innovadora de creación de conocimientos era prácticamente invisible. Por el contrario, en el caso de las empresas aglomeradas en sectores vinculados indirectamente a los RRNN, los resultados sugieren que aun en los momentos más oscuros de la destrucción de las capacidades innovadoras de finales de los años ochenta y principios de los noventa, el sector agrícola sostenía a un grupo de proveedores de químicos y maquinaria que constituía un locus de empresas innovadoras aglomeradas a la vanguardia de la actividad innovadora como creadoras de conocimientos dentro del sector manufacturero argentino.

Resulta interesante, además, complementar los datos anteriores con otros que den cuenta de la evolución en el comportamiento innovador de las empresas durante el período analizado de 1992 a 2004. En el cuadro 8 se muestran los resultados relativos a los indicadores analizados. En el transcurso de más de una década, las firmas innovadoras aglomeradas tanto en sectores directamente vinculados como indirectamente vinculados a los RRNN se destacaron por la profundización en la intensidad de inversión en actividades de I+D. En particular, las primeras incrementaron la intensidad en este gasto a un ritmo casi tres veces más rápido que la media del sector manufacturero, es decir, bastante más que las

CUADRO 8

Argentina: cambios en el comportamiento innovador del sector manufacturero, 1992-2004

\begin{tabular}{|c|c|c|}
\hline \multirow{3}{*}{ Tipos de empresa } & \multicolumn{2}{|c|}{$\begin{array}{c}\text { Cambio promedio anual } \\
1992-2004\end{array}$} \\
\hline & Intensidad en I+D & Inversión de capital \\
\hline & \multicolumn{2}{|c|}{$\begin{array}{l}\text { Valores medios por empresa } \\
\text { (en porcentajes) }\end{array}$} \\
\hline 1. Empresas innovadoras aglomeradas en sectores: & 17,12 & $-4,29$ \\
\hline directamente vinculados a los RRNN & 31,84 & $-0,38$ \\
\hline indirectamente vinculadas a los RRNN & 16,25 & $-8,96$ \\
\hline 2. Todas las demás empresas innovadoras & 8,69 & $-7,40$ \\
\hline 3. Empresas en industrias "de alta tecnología" & 10,83 & $-10,59$ \\
\hline 4. Filiales extranjeras & 11,02 & $-14,95$ \\
\hline 5. Empresas no innovadoras & 23,41 & $-7,50$ \\
\hline 6. Todas las empresas & 11,57 & $-7,34$ \\
\hline
\end{tabular}

Fuente: elaboración propia sobre la base de la Encuesta sobre la Conducta Tecnológica de las Empresas Industriales Argentinas (1992-1996) y la Encuesta Nacional a Empresas sobre Innovación, Investigación y Desarrollo y Tecnologías de la Información y de las Comunicaciones 2002-2004 en la Argentina.

RRNN: recursos naturales.

I+D: investigación y desarrollo. 
empresas no innovadoras que, junto con este grupo de firmas, eran las que menos invertían en I+D al principio del período. Con respecto a las empresas innovadoras aglomeradas en sectores indirectamente vinculados a actividades basadas en RRNN, estas aumentaron la intensidad del gasto en I+D a un ritmo más acelerado que el resto de los grupos que tenían niveles comparables de intensidad de gastos en I+D a principios del período (como es el caso de las empresas en sectores de alta tecnología). Las firmas aglomeradas no vinculadas a los RRNN, en cambio, fueron las únicas que mostraron tasas negativas de incremento del gasto en actividades de I+D.

En el período 1992-2004, las inversiones en bienes de capital para la innovación se redujeron en todos los grupos de firmas considerados. Sin embargo, las empresas innovadoras aglomeradas en sectores vinculados a los RRNN fueron las que menos redujeron las inversiones en este rubro como porcentaje de sus ventas. En particular, las empresas innovadoras aglomeradas en sectores directamente vinculados a los RRNN fueron las únicas que prácticamente mantuvieron la inversión en bienes de capital para la innovación en los niveles de principios del período, aunque sus ventas aumentaron sustancialmente.

En suma, tomados en conjunto con respecto a todo el período analizado, los dos grupos de empresas innovadoras aglomeradas en sectores vinculados a actividades basadas en la explotación de RRNN constituían — según sus esfuerzos relativos en actividades innovadoras-un locus importante de capacidad de creación y uso de conocimientos en la industria manufacturera argentina. Esto aparece como sustancial y potencialmente importante sobre todo durante un período de gran inestabilidad y turbulencia como el considerado.

\section{Las empresas innovadoras aglomeradas en el Brasil}

En el Brasil, entre las empresas innovadoras aglomeradas (vinculadas y no vinculadas a actividades basadas en RRNN) se aprecia una mayor participación de grupos empresariales nacionales y una menor incidencia de empresas independientes nacionales con respecto al total del sector manufacturero. Sin embargo, existen diferencias sustantivas en la composición del grupo de empresas innovadoras vinculadas y no vinculadas a actividades basadas en RRNN. Entre las primeras se destaca una alta presencia relativa de filiales de compañías extranjeras con el $44 \%$ de las firmas, mientras que entre las segundas las empresas foráneas son poco significativas (un 5\%). Además, los grupos empresariales nacionales y las empresas nacionales independientes son más significativos entre las empresas vinculadas a los RRNN ( $45 \%$ y $40 \%$, respectivamente) que entre las firmas no vinculadas a actividades basadas en RRNN (23\% y $33 \%$, respectivamente), según puede apreciarse en el cuadro 9. En cuanto a su magnitud, las empresas innovadoras aglomeradas se destacan en general por un tamaño relativo mayor respecto del sector manufacturero en general, de las empresas innovadoras aisladas y de las no innovadoras. El $87 \%$ de las empresas innovadoras aglomeradas en industrias vinculadas a los RRNN y el $100 \%$ de las empresas aglomeradas no vinculadas a los RRNN poseen más de 200 empleados. En relación con el total del sector manufacturero, las empresas de ese tamaño representan el 56\% de las firmas (véase el cuadro 10).

Con respecto al comportamiento innovador, de acuerdo con las cifras presentadas en el cuadro 11, en el año 2001 las empresas innovadoras aglomeradas tomadas en conjunto realizaban más esfuerzos en I+D en relación con sus ventas $(0,91 \%)$ que las no innovadoras $(0,36 \%)$ y que las filiales de compañías multinacionales $(0,79 \%)$, pero efectuaban menos esfuerzos relativos respecto del resto de las empresas innovadoras $(1,30 \%)$ y de las empresas en actividades de alta tecnología $(2,06 \%)$. Conviene destacar que las empresas innovadoras aglomeradas en sectores indirectamente vinculados a actividades basadas en RRNN duplicaron con creces los esfuerzos en actividades de I+D respecto de aquellas firmas aglomeradas no vinculadas a actividades basadas en RRNN $(1,17 \%$ y $0,52 \%$, respectivamente). Además, las empresas innovadoras aglomeradas vinculadas a los RRNN como proveedoras se encuentran junto con aquellas de alta tecnología entre las empresas que más esfuerzos en actividades de I+D han realizado en relación con sus ventas en el año analizado.

Cuando se observa la evolución de los indicadores de las actividades de innovación de las empresas, aparecen otros resultados interesantes. Entre 2000 y 2005, en un contexto generalizado de reducción de la intensidad de inversión en actividades de I+D en el sector manufacturero brasileño, las empresas innovadoras aglomeradas fueron las únicas que incrementaron anualmente los esfuerzos en estas actividades (véase el cuadro 12). Entre estas se destacan las empresas innovadoras aglomeradas no vinculadas a actividades basadas en RRNN, que aumentaron su inversión en actividades de I+D con una intensidad del $19,18 \%$. Otros esfuerzos innovadores, tales como la adquisición de conocimiento incorporado en bienes de capital, fueron en general negativos durante el período analizado. Sin embargo, las empresas innovadoras aglomeradas vinculadas a los RRNN mostraron la menor caída en la inversión en bienes de capital respecto del resto de las firmas $(-4,64 \%$ anual). 
Brasil: empresas innovadoras por tipo de titularidad (Cantidad y porcentajes)

\begin{tabular}{|c|c|c|c|c|c|}
\hline & $\begin{array}{c}\text { Empresas } \\
\text { innovadoras } \\
\text { aglomeradas en } \\
\text { industrias vinculadas } \\
\text { a los RRNN }\end{array}$ & $\begin{array}{c}\text { Empresas } \\
\text { innovadoras } \\
\text { aglomeradas } \\
\text { no vinculadas } \\
\text { a los RRNN }\end{array}$ & $\begin{array}{c}\text { Empresas } \\
\text { innovadoras } \\
\text { aisladas }\end{array}$ & $\begin{array}{c}\text { Empresas no } \\
\text { innovadoras en } \\
\text { todas las industrias }\end{array}$ & Total \\
\hline & \multicolumn{5}{|c|}{ Número de empresas y proporciones de los totales de las columnas (en porcentajes) } \\
\hline Grupos empresariales nacionales & $\begin{array}{c}7 \\
(23)\end{array}$ & $\begin{array}{c}9 \\
(45)\end{array}$ & $\begin{array}{c}29 \\
(25)\end{array}$ & $\begin{array}{l}486 \\
(13)\end{array}$ & $\begin{array}{l}522 \\
(13)\end{array}$ \\
\hline Empresas nacionales independientes & $\begin{array}{c}10 \\
(33)\end{array}$ & $\begin{array}{c}8 \\
(40)\end{array}$ & $\begin{array}{c}43 \\
(37)\end{array}$ & $\begin{array}{c}2677 \\
(72)\end{array}$ & $\begin{array}{c}2730 \\
(71)\end{array}$ \\
\hline Filiales de empresas multinacionales & $\begin{array}{c}13 \\
(44)\end{array}$ & $\begin{array}{c}3 \\
(5)\end{array}$ & $\begin{array}{c}45 \\
(38)\end{array}$ & $\begin{array}{l}559 \\
(15)\end{array}$ & $\begin{array}{l}617 \\
(16)\end{array}$ \\
\hline Total & $\begin{array}{c}30 \\
(100)\end{array}$ & $\begin{array}{c}20 \\
(100)\end{array}$ & $\begin{array}{c}117 \\
(100)\end{array}$ & $\begin{array}{l}3722 \\
(100)\end{array}$ & $\begin{array}{l}3889 \\
(100)\end{array}$ \\
\hline
\end{tabular}

Fuente: elaboración propia sobre la base de la Encuesta de Innovación Tecnológica (PINTEC) 2000 y 2005.

RRNN: recursos naturales.

Brasil: empresas por tamaño (Cantidad y porcentajes)

\begin{tabular}{|c|c|c|c|c|c|}
\hline & $\begin{array}{c}\text { Empresas } \\
\text { innovadoras } \\
\text { aglomeradas en } \\
\text { industrias vinculadas } \\
\text { a los RRNN }\end{array}$ & $\begin{array}{c}\text { Empresas } \\
\text { innovadores } \\
\text { aglomeradas } \\
\text { no vinculadas } \\
\text { a los RRNN }\end{array}$ & $\begin{array}{c}\text { Empresas } \\
\text { innovadoras } \\
\text { aisladas }\end{array}$ & $\begin{array}{c}\text { Empresas no } \\
\text { innovadoras en } \\
\text { todas las industrias }\end{array}$ & Total \\
\hline Pymes & $\begin{array}{c}4 \\
(13)\end{array}$ & $\begin{array}{c}0 \\
(0)\end{array}$ & $\begin{array}{c}17 \\
(15)\end{array}$ & $\begin{array}{c}1680 \\
(45)\end{array}$ & $\begin{array}{c}1701 \\
(44)\end{array}$ \\
\hline Grandes empresas & $\begin{array}{c}26 \\
(87)\end{array}$ & $\begin{array}{c}20 \\
(100)\end{array}$ & $\begin{array}{l}100 \\
(85)\end{array}$ & $\begin{array}{c}2042 \\
(55)\end{array}$ & $\begin{array}{c}2168 \\
(56)\end{array}$ \\
\hline Total & $30(100)$ & $20(100)$ & $117(100)$ & $3722(100)$ & 3889 (100) \\
\hline
\end{tabular}

Fuente: elaboración propia sobre la base de la Encuesta de Innovación Tecnológica (PINTEC) 2000 y 2005.

RRNN: recursos naturales.

Nota: pymes: pequeñas y medianas empresas $(<200$ empleados) y grandes empresas $(\geq 200$ empleados).

CUADRO 11

Brasil: comportamiento innovador del sector manufacturero, 2000

Tipo de empresa

\begin{tabular}{|c|c|}
\hline Intensidad & Inversión de capital \\
\hline \multicolumn{2}{|c|}{$\begin{array}{l}\text { Valores medios por empresa } \\
\text { (en porcentajes) }\end{array}$} \\
\hline 0,91 & 2,09 \\
\hline 1,17 & 1,74 \\
\hline 0,52 & 2,61 \\
\hline 1,30 & 3,56 \\
\hline 2,06 & 2,01 \\
\hline 0,79 & 6,03 \\
\hline 0,36 & 4,72 \\
\hline 0,97 & 3,89 \\
\hline
\end{tabular}

Fuente: elaboración propia sobre la base de la Encuesta de Innovación Tecnológica (PINTEC) 2000 y 2005.

RRNN: recursos naturales.

I+D: investigación y desarrollo. 
CUADRO 12

Brasil: cambios en el comportamiento innovador del sector manufacturero, 2000-2005

\begin{tabular}{|c|c|c|}
\hline \multirow[b]{2}{*}{ Tipo de empresa } & Intensidad en $\mathrm{I}+\mathrm{D}$ & Inversión de capital \\
\hline & \multicolumn{2}{|c|}{$\begin{array}{l}\text { Valores medios por empresa } \\
\quad(\text { en porcentajes })\end{array}$} \\
\hline 1. Empresas innovadoras aglomeradas: & 5,72 & $-12,99$ \\
\hline indirectamente vinculadas a los RRNN & 1,50 & $-4,64$ \\
\hline no vinculadas a industrias basadas en RRNN & 16,15 & $-26,95$ \\
\hline 2. Todas las demás empresas innovadoras & $-14,84$ & $-10,80$ \\
\hline 3. Empresas en industrias "de alta tecnología" & $-1,22$ & $-8,66$ \\
\hline 4. Filiales extranjeras (de compañías multinacionales) & $-3,89$ & $-7,34$ \\
\hline 5. Empresas no innovadoras & $-2,14$ & $-27,32$ \\
\hline 6. Todas las empresas & $-8,23$ & $-12,72$ \\
\hline
\end{tabular}

Fuente: elaboración propia sobre la base de la Encuesta de Innovación Tecnológica (PINTEC) 2000 y 2005.

RRNN: recursos naturales.

I+D: investigación y desarrollo.

En suma, las empresas innovadoras aglomeradas brasileñas (vinculadas y no vinculada a actividades basadas en RRNN a través de relaciones de insumoproducto), de acuerdo con los indicadores analizados, constituyen un locus de innovación relevante en el sector manufacturero brasileño. Resulta interesante constatar que estas empresas se destacan durante todo el período estudiado por realizar los mayores esfuerzos en actividades de innovación (como la I+D) que son poco observables en el sector manufacturero de los países de la región.

\section{VI}

\section{Discusión y reflexión final}

En este trabajo se estudió la evolución de la capacidad innovadora en los sectores manufactureros argentino y brasileño durante un período caracterizado por alta inestabilidad económica y cambios en las políticas económicas en ambos países. En muchos estudios se documentó la destrucción de empresas y capacidades tecnológicas que primaron durante este lapso en la región. Se investigó el posible resurgimiento de capacidades innovadoras y su distribución sectorial. En el análisis se utilizaron datos intertemporales de las encuestas de innovación de la Argentina y el Brasil, cubriendo el período de 1992 a 2004 en el primer país y de 2001 a 2005 en el segundo. Los resultados no son definitivos puesto que se basan en el análisis de la evidencia existente, que es imperfecta y fragmentada. Sin embargo, permiten algunas reflexiones y sugerencias potencialmente importantes para la investigación futura. En particular, vale la pena mencionar y discutir dos de estas.

Primero, se encuentra un número significativo de empresas aglomeradas en un grupo reducido de sectores, las que se identificaron con la vanguardia de la actividad innovadora e incrementaron ostensiblemente sus esfuerzos innovadores durante el período bajo estudio. La evidencia analizada no sugiere que estas empresas sean líderes mundiales, siendo una indicación de aquello los bajos gastos en I+D que registran, muy lejanos de los estándares mundiales; sin embargo, en términos relativos, se diferencian del resto de las empresas en cada país y, en tal sentido, resulta interesante analizar sus características.

Segundo, conviene recalcar que gran parte de esas empresas innovadoras se aglomeran en algunas actividades que no son las que típicamente se esperaría. En la Argentina se destacaron por su evolución las empresas aglomeradas en sectores usuarios de RRNN, en el Brasil, las aglomeradas en sectores tradicionales, y en ambos países las vinculadas a los RRNN como proveedoras y aglomeradas en sectores protegidos por regímenes especiales. Vale la pena reflexionar sobre los dos primeros tipos: i) las empresas aglomeradas 
en sectores vinculados a los RRNN, en particular las usuarias de estos, y ii) las empresas aglomeradas en sectores tradicionales.

i) Cercanía a los RRNN: las industrias cercanas a los RRNN son, en general, consideradas de baja oportunidad tecnológica. Desde la década de 1950, en la literatura relacionada con la temática del desarrollo se ha sostenido que las actividades basadas en la explotación y procesamiento de los RRNN tienen características que no favorecen el aprovechamiento de las ventajas del cambio tecnológico y el agregado de valor en comparación con otras actividades, como las manufacturas industriales (Prebisch, 1949; Singer, 1950; Nurske, 1958). De la misma manera, en la literatura de innovación se ha identificado a las industrias manufactureras estrechamente vinculadas a los RRNN como de baja oportunidad tecnológica; de hecho, son clasificadas como de "baja tecnología" en las taxonomías de industrias (OCDE, 1997). En consecuencia, la recomendación típica de política para los países con una marcada especialización en RRNN ha sido que incentiven el desarrollo de otros sectores asociados a mayores oportunidades, en términos de valor agregado, como por ejemplo aquellas actividades que se aglutinan en los llamados segmentos de "alta tecnología". No obstante, recientemente algunos autores han comenzado a enfatizar que los sectores basados en la explotación de RRNN están intensificando su uso de conocimiento y, por lo tanto, abriendo oportunidades para un mayor aprendizaje e innovación, y asimismo para alentar el desarrollo de sectores innovadores dinámicos conexos (Marín, Navas-Alemán y Pérez, en prensa; Pérez, 1999, 2001 y 2010; Kaplinsky y Fitter, 2004). Ello se debe, en gran medida, a que las condiciones históricas han variado fundamentalmente junto con cambios en la demanda y la difusión de nuevos paradigmas tecnológicos. La demanda de alimentos, materias primas y energía se ha intensificado en los últimos años debido a la creciente globalización de los mercados (y la incorporación de China al mercado mundial), incrementándose también la demanda por variedad y calidad (como el aumento en la demanda de alimentos gourmet, de productos más amigables con el medioambiente o más seguros). Esto está abriendo oportunidades que antes no existían para invertir en conocimiento y generar variedad en conjunto con los RRNN. A su vez, la difusión de las nuevas tecnologías, como la biotecnología y la nanotecnología, está multiplicando las posibilidades de innovación y diferenciación, así como las tecnologías de la información y las comunicaciones (TIC) están favoreciendo y facilitando la incorporación de zonas remotas de producción al mercado mundial (Von Tunzelmann y Acha, 2005, Marín, Navas-Alemán y Pérez, en prensa). Los resultados aquí presentados pueden estar empezando a captar estos fenómenos.

ii) Sectores tradicionales: el caso del Brasil, se observan además áreas de creación de ventajas competitivas en sectores no estrechamente vinculados a los RRNN, pero tradicionales como la fabricación de calzado o de productos de plástico. Ello puede obedecer también a la penetración de nuevas tecnologías en sectores usualmente considerados de baja y media tecnología (Von Tunzelmann y Acha, 2005) y a otros fenómenos emergentes que la literatura ha empezado a identificar. En estudios recientes se resalta, de hecho, que los sectores tradicionales o de baja/media tecnología no necesariamente aglomeran empresas no innovadoras (Kirner, Kinkel y Jaeger, 2009; Hirsch-Kreinsen, 2008). Esto se debe a que los productos que se elaboraban típicamente mediante tecnologías antiguas o ya maduras, están empezando a producirse con tecnologías radicalmente nuevas o características de otra industria (Kirner, Kinkel y Jaeger, 2009). La aplicación de la biotecnología al procesamiento de alimentos es un claro ejemplo de cómo un sector considerado de baja intensidad tecnológica ha comenzado a utilizar tecnologías identificadas como de alto nivel y se está dinamizando. Se argumenta que esta difusión tecnológica horizontal entre sectores tendería a borrar la identificación de industrias con productos y tecnologías, y a tornar menos útiles ciertas clasificaciones sectoriales vastamente difundidas y utilizadas.

Tomados en su conjunto, estos resultados sugieren que las taxonomías sectoriales existentes no parecen ser del todo relevantes, o que no estarían captando fenómenos recientes como la intensificación de la actividad innovadora en sectores tradicionalmente considerados de baja tecnología, como los vinculados directamente a los RRNN y los tradicionales. También sugieren que las políticas de desarrollo industrial tendientes a atraer ganadores (picking winners), centradas en la identificación y fortalecimiento de sectores aislados, no parecen las más adecuadas en el contexto actual. Por el contrario, en términos de política industrial parece más prometedor considerar la interacción e interdependencia entre sectores (Hansen y Winther, 2010). Se sabe, por ejemplo, que las empresas en industrias usualmente consideradas de baja y media tecnología, además de 
ser las más relevantes en términos de generación de producto, empleo y capital invertido, son las principales consumidoras de innovaciones de alta tecnología (Robertson, Pol y Carroll, 2003). Consecuentemente, el crecimiento de actividades de alta tecnología depende, en gran medida, del crecimiento de otras actividades conexas generalmente menos intensivas en I+D (Hauknes y Knell, 2009; Robertson y Patel, 2007). Esta interacción debe considerarse en las estrategias de desarrollo de la región, evitando un excesivo sesgo hacia el incentivo de sectores de alta tecnología, sin contemplar cómo estos se vinculan e interaccionan con otros sectores productivos.

Finalmente, se señalan algunos interrogantes, limitaciones y sugerencias para investigaciones futuras:

Cabe destacar algunas limitaciones de los indicadores utilizados. En primer lugar, los indicadores que miden el proceso innovador se focalizan casi exclusivamente en el sector manufacturero, impidiendo indagar acerca del patrón de innovación en otras actividades importantes para la región, como la actividad primaria o el sector de servicios. Asimismo, los indicadores de resultado del proceso innovador disponibles presentan limitaciones para captar de modo acertado la dinámica de innovación en estos países. Por una parte, las patentes son un indicador ampliamente aceptado y difundido en los países más desarrollados, pero limitado como indicador, dado que la mayor parte del proceso innovador de las firmas de la región consiste en innovaciones de tipo incremental y adaptativo o de bajo grado de novedad, lo que impide la utilización del sistema de patentes para su protección. Por otra parte, otros indicadores de resultado de innovación disponibles, como el utilizado en este trabajo, se basan en la subjetividad de la respuesta que provee la empresa encuestada respecto del logro o no de innovaciones en un cierto período, y del grado de novedad de dicha innovación. En la literatura se reconoce que ello puede conducir a una sobreestimación del nivel innovador de las firmas. Por lo tanto, las limitaciones de los indicadores utilizados y de los datos disponibles demandan cautela a la hora de interpretar los resultados y hacer generalizaciones definitivas.

Segundo, en este trabajo se habla de resurgimiento, pero los indicadores de esfuerzos de innovación, como el de I+D, son en algunos casos no esperables, extremadamente bajos. Cabe preguntarse, entonces, si son bajos porque con los indicadores disponibles no se pudieron identificar verdaderos innovadores, o porque los innovadores más significativos se encuentran en sectores de RRNN y tradicionales donde se innova de otra manera (la innovación incremental y de procesos, y los esfuerzos ingenieriles son los más importantes). A futuro, se debiera saber más acerca de las características de la innovación en cada sector, con el objeto de poder identificar indicadores que sirvan para captar diferencias en intensidad de esfuerzos y resultados independientemente de las características de los sectores. Ello permitirá de seguro arrojar más luz sobre los interrogantes planteados en este estudio.

ANEXO

Identificación de sectores relacionados con los recursos naturales (RRNN)

Se identifican dos tipos de industrias relacionadas con la producción de RRNN: las consumidoras y las proveedoras. La clasificación se realizó empleando índices que miden la proximidad relativa a actividades basadas en la explotación de RRNN ${ }^{12}$. En dichos índices se utiliza información acerca del valor de las transacciones entre industrias que surge de las matrices de insumo-producto (MIP) de cada país.

\footnotetext{
12 Sobre la base de la matriz insumo-producto (MIP), los sectores de RRNN son: cultivo de cereales, oleaginosas y forrajeras; cultivo de hortalizas, legumbres, flores y plantas ornamentales; cultivo de frutas y nueces; cultivos industriales; producción de semillas; crianza de ganado y producción de leche, lana y pelos; producción de granja; servicios agropecuarios; caza; silvicultura y extracción de madera; pesca; extracción de petróleo, gas, carbón y uranio; extracción de minerales metalíferos; extracción de otros minerales.
}

Debido a que las actividades de la MIP no se corresponden unívocamente con la Clasificación Internacional Industrial Uniforme de todas las actividades económicas (CIIU) a tres dígitos, las transacciones de cada sector se ponderaron por la suma del valor de producción de los sectores a tres dígitos que corresponden a una misma actividad de la MIP.

El primer índice es la proporción de compras a los sectores de RRNN hechas por cada industria manufacturera sobre el total de compras realizadas por ese sector. Cuanto mayor es la proporción, mayor es la proximidad directa a los RRNN.

Para cada sector $j$ se aplicó el siguiente procedimiento:

$$
\text { Índice RRNN Cons }{ }_{j}=\frac{\operatorname{suma}\left(M I P_{m=R R N N, n=j}\right)}{\operatorname{suma}\left(M I P_{m, n=j}\right)}
$$


Un procedimiento análogo se aplicó para crear el segundo índice utilizado para las industrias proveedoras de RRNN. Cuanto mayor el valor del índice, mayor su proximidad indirecta con los RRNN:

$$
\text { Índice RRNN Sup } j=\frac{\operatorname{suma}\left(\operatorname{MIP} P_{m=R R N N, n=j}^{T}\right)}{\operatorname{suma}\left(\operatorname{MIP}_{m, n=j}^{T}\right)}
$$

Los sectores identificados como directamente relacionados con los RRNN son aquellos cuyos índices como consumidores de dichos recursos muestran valores por sobre la media; y los sectores clasificados como indirectamente relacionados con los recursos naturales son aquellos cuyo índice como proveedores de RRNN sobrepasa la media. Los sectores con valores superiores a la media en ambos grupos se clasificaron en aquel respecto del cual mostraban un mayor valor.

\section{Bibliografía}

Amsden, A.H. (1989), Asia's Next Giant: South Korea and Late Industrialization, Nueva York, Oxford University Press.

Baer, W. (2001), The Brazilian Economy: Growth and Development, Greenwood Publishing Group.

(1972), "Import substitution and industrialization in Latin America: experiences and interpretations", Latin American Research Review, vol. 7, $\mathrm{N}^{\circ} 1$, Asociación de Estudios Latinoamericanos.

(1970), Siderurgia e desenvolvimento brasileiro, Río de Janeiro, Zahar Editores.

Barros de Castro, A. (2003), "El segundo catch-up brasileño: Características y limitaciones", Revista de la CEPAL, $\mathrm{N}^{\circ} 80$ (LC/G.2204-P), Santiago de Chile, agosto.

Chudnovsky, D., A. López y G. Pupato (2006), "Innovation and productivity in developing countries: a study of Argentine manufacturing firms' behavior (1992-2001)", Research Policy, vol. 35, $\mathrm{N}^{\circ} 2$, Amsterdam, Elsevier.

Cimoli, M. y J. Katz (2003), "Structural reforms, technological gaps and economic development: a Latin American perspective", Industrial and Corporate Change, vol. 12, $\mathrm{N}^{\circ} 2$, Oxford, Oxford University Press.

Dahlman, C. (1984), "Foreign technology and indigenous technological capability in Brazil", Technological Capability in the Third World, M. Fransman y K. King, Londres, Macmillan.

Dahlman, C. y F. Fonseca (1987), "From technological dependence to technological development: the case of Usiminas steelplant in Brazil", Technology Generation in Latin American Manufacturing Industries, J. Katz (ed.), Londres, Macmillan.

Dahlman, C. y C. Frischtak (1990), "National systems supporting technical advance in industry: the Brazilian experience", National Innovation Systems. A Comparative Analysis, R. Nelson (ed.), Oxford, Oxford University Press.

Erber, F., E. Guimarães y J. Tavares Araújo Jr. (1974), Absorção e criação de tecnologia na indústria de bens de capital, Río de Janeiro, Financiadora de Estudios y Proyectos (FINEP).

Ferraz, J.C., D. Kupfer y L. Haguenauer (1996), "El desafío competitivo para la industria brasileña", Revista de la CEPAL, $\mathrm{N}^{\circ} 58$ (LC/G.1916-P), Santiago de Chile, abril.

Ferraz, J.C., D. Kupfer y F. Serrano (1999), "Macro/micro interactions: economic and institutional uncertainties and structural change in the Brazilian industry", Oxford Development Studies, vol. 27, $\mathrm{N}^{\circ}$ 3, Taylor \& Francis.

Ferraz, J.C., D. Kupfer y M. Iootty (2004), "Competitividad industrial en Brasil 10 años después de la liberalización”, Revista de la CEPAL, $\mathrm{N}^{\circ} 82$ (LC/G.2220-P), Santiago de Chile, abril.

Hansen, T. y L. Winther (2010), "Innovation, regional development and relations between high- and low-tech industries", documento presentado en la octava Conferencia Europea sobre estudios urbanos y regionales, Viena.
Harabi, N. (1995), "Appropriability of technical innovations: an empirical analysis", Research Policy, vol. 24, № 6, Amsterdam, Elsevier.

Hauknes, J. y M. Knell (2009), "Embodied knowledge and sectoral linkages: An input-output approach to the interaction of highand low-tech industries", Research Policy, vol. 38, $\mathrm{N}^{\circ} 3$, Amsterdam, Elsevier

Hirsch-Kreinsen, H. (2008), "'Low-tech' innovations", Industry and Innovation, vol. $15, \mathrm{~N}^{\circ} 1$, Taylor \& Francis

Hobday, M. (1995), Innovation in East Asia: The Challenge to Japan, Aldershot, Edward Elgar.

Kaplinsky, R. y R. Fitter (2004), "Technology and globalisation: who gains when commodities are decommodified?", International Journal of Technology and Globalization, vol. 1, $\mathrm{N}^{\circ} 1$, Inderscience Publishers.

Katz, J. (2007), "Cycles of creation and destruction of production capacity and the development of new export and technological capabilities in Latin America", Can Latin American Firms Compete?, T. Grosse y L. Mesquita (eds.), Oxford Scholarship Online.

(2001), "Structural reforms and technological behaviour. The sources and nature of technological change in Latin America in the 1990s", Research Policy, vol. 30, º 1, Amsterdam, Elsevier.

(1987), "Domestic technology generation in LDCs: a review of research findings", Technology Generation in Latin American Manufacturing Industries, Londres, Macmillan.

(1972), "Importación de tecnología, aprendizaje local e industrialización dependiente", Documento de trabajo, Buenos Aires, Instituto Torcuato di Tella.

Katz, J. y N.A. Bercovich (1993), "National systems of innovation supporting technical advance in industry: the case of Argentina", National Innovation Systems: A Comparative Analysis, R.R. Nelson (ed.), Nueva York, Oxford University Press.

Katz, J. y B. Kosacoff (2000), "Import-substituting industrialisation in Argentina, 1940-1980: its achievements and shortcomings", Industrialisation and the State in Latin-America: the Post War Years, E. Cárdenas, J.A. Ocampo y R. Thorp (eds.), Basingstoke.

(1989), "El proceso de industrialización en la Argentina: Evolución, retroceso y prospectiva", Buenos Aires, Centro Editor de América Latina.

Kim, L. (1997), Imitation to Innovation: The Dynamics of Korea's Technological Learning, Boston, Massachusetts, Harvard Business School Press.

Kirner, E., S. Kinkel y A. Jaeger (2009), "Innovation paths and the innovation performance of low-technology firms - An empirical analysis of German industry", Research Policy, vol. 38, № 3, Amsterdam, Elsevier. 
Kosacoff, B. (2000a), Corporate Strategies under Structural Adjustment in Argentina: Responses by Industrial Firms to a New Set of Uncertainties, Basingstoke, Macmillan.

(2000b), El desempeño industrial argentino más allá de la sustitución de importaciones, Buenos Aires, Oficina de la CEPAL en Buenos Aires.

(1996), "Estrategias empresariales en la transformación industrial argentina", Documento de Trabajo, No 67 (LC/ BUE/L.150), Santiago de Chile, Comisión Económica para América Latina y el Caribe (CEPAL).

Kulfas, M., F. Porta y A. Ramos (2002), "Inversión extranjera y empresas transnacionales en la economía argentina", Serie Estudios y Perspectivas, $\mathrm{N}^{\circ} 10$ (LC/L.1766-P), Buenos Aires, Oficina de la CEPAL en Buenos Aires. Publicación de las Naciones Unidas, $\mathrm{N}^{\circ}$ de venta: S.02.II.G.80.

Levin, R.C. y otros (1987), "Appropriating the returns from industrial research and development", Brookings Papers on Economic Activity, vol. 18, $\mathrm{N}^{\circ} 3$, Washington, D.C., The Brookings Institution.

López, A. (1996), "Las ideas evolucionistas en economía: Una visión de conjunto", Revista Buenos Aires. Pensamiento Económico, $\mathrm{N}^{\circ} 1$, Buenos Aires.

Marín, A. y M. Bell (2012), "Argentina", Innovative Firms in Emerging Market Countries, E. Amann y J. Cantwell (eds.), Oxford, Oxford University Press.

Marín A., L. Navas-Alemán y C. Pérez (en prensa), "Natural Resource Industries as a Platform for the Development of Knowledge Instensive Industries",

Mathews, J. y D.-S. Cho (2000), Tiger Technology: The Creation of a Semiconductor Industry in East Asia, Cambridge, Cambridge University Press.

Naciones Unidas (1969), "The manufacture of machine tools in Brazil”, Development of Metalworking Industries in Developing Countries, Nueva York.

Nurske, R. (1958), "Trade fluctuations and buffer policies of lowincome countries", Kyklos, vol. 11, $\mathrm{N}^{\circ}$ 2, Wiley.

ocDE (Organización de Cooperación y Desarrollo Económicos) (2005), Manual de Oslo: Guía para la recogida e interpretación de datos sobre innovación, París.

(1997), "Revision of the high-technology sector and product classification", OECD Science, Technology and Industry Working Papers, N 1997/02, París.
Patel, P. (2000), "Technological indicators of performance", From Knowledge Management to Strategic Competence: Measuring Technological, Market and Organisational Innovation, J. Tidd (ed.), Londres, Imperial College Press.

Pérez, C. (2010), "Dinamismo tecnológico e inclusion social en América Latina: Una estrategia de desarrollo productivo basada en los recursos naturals", Revista CEPAL, $\mathrm{N}^{\circ} 100$, (LC/G.2442-P), Santiago de Chile, abril.

(2001), "Cambio tecnológico y oportunidades de desarrollo como blanco móvil", Revista de la CEPAL, $\mathrm{N}^{\circ} 75$ (LC/G.2150-P), Santiago de Chile, diciembre.

(1999), "Cambio de patrón tecnológico y oportunidades para el desarrollo sustentable", Colección Ideas para el Diálogo, $\mathrm{N}^{\circ}$ 3, Caracas, Biblioteca Nacional de Venezuela.

Prebisch, R. (1949), El desarrollo económico de la América Latina y algunos de sus principales problemas (E/CN.12/89), Naciones Unidas.

Robertson, P.L., E. Pol y P. Carroll (2003), "Receptive capacity of established industries as a limiting factor in the economy's rate of innovation", Industry and Innovation, vol. 10, $\mathrm{N}^{\circ} 4$, Taylor \& Francis.

Robertson, P.L. y P.R. Patel (2007), "New wine in old bottles technological diffusion in developed economies", Research Policy, vol. 36, $\mathrm{N}^{\circ}$ 5, Amsterdam, Elsevier.

Rocha, F. y D. Kupfer (2002), "Structural changes and specialization in Brazilian industry: the evolution of leading companies and the M\&A process", The Developing Economies, vol. 40, № 4 , Tokio, Instituto de las Economías en Desarrollo, diciembre.

Scherer, F.M. (1983), "The propensity to patent", International Journal of Industrial Organization, vol. 1, $\mathrm{N}^{\circ} 1$, Amsterdam, Elsevier.

Singer, H. (1950), "The distribution of gains between investing and borrowing countries", American Economic Review, vol. 40, $\mathrm{N}^{\circ}$ 2, Nashville, Tennessee, American Economic Association.

Teitel, S. (1981), "Towards an understanding of technical change in semi-industrialized countries", Research Policy, vol. 10, $\mathrm{N}^{\circ} 2$, Amsterdam, Elsevier.

Teitel, S. y F. Thoumi (1987), "De la sustitución de importaciones a las exportaciones: La experiencia de las exportaciones manufactureras de la Argentina y el Brasil", Desarrollo Económico, vol. 27, $\mathrm{N}^{\circ} 105$, Buenos Aires, Instituto de Desarrollo Económico y Social.

Von Tunzelmann, N. y V. Acha (2005), "Innovation in 'low-tech' industries", The Oxford Handbook of Innovation, J. Fageberg, D. Mowery y R. Nelson (eds.), Oxford, Oxford University Press. 